\title{
Analytical Expression for the Concentration of Substrate and Product in Immobilized Enzyme System in Biofuel/Biosensor
}

\author{
R. Malini Devi', 0. M. Kirthiga², L. Rajendran² \\ ${ }^{1}$ Department of Mathematics, The Standard Fireworks Rajaratnam College for Women, Sivakasi, India \\ ${ }^{2}$ Department of Mathematics, The Madura College, Madurai, India \\ Email: rajssms@rediffmail.com
}

Received 21 November 2014; accepted 19 June 2015; published 23 June 2015

Copyright (C) 2015 by authors and Scientific Research Publishing Inc.

This work is licensed under the Creative Commons Attribution International License (CC BY). http://creativecommons.org/licenses/by/4.0/

(c) (7) Open Access

\begin{abstract}
In this paper, an approximate analytical method to solve the non-linear differential equations in an immobilized enzyme film is presented. Analytical expressions for concentrations of substrate and product have been derived for all values of dimensionless parameter. Dimensionless numbers that can be used to study the effects of enzyme loading, enzymatic gel thickness, and oxidation/ reduction kinetics at the electrode in biosensor/biofuel cell performance were identified. Using the dimensionless numbers identified in this paper, and the plots representing the effects of these dimensionless numbers on concentrations and current in biosensor/biofuel cell are discussed. Analytical results are compared with simulation results and satisfactory agreement is noted.
\end{abstract}

\section{Keywords}

Michaelis-Menten Kinetics, Biofuel and Biosensor, Homotopy Perturbation Method, Immobilized Enzyme Systems

\section{Introduction}

Biosensors and biofuel cells are commonly used for industrial, environmental and medical applications. However there are no clear guidelines for the design of electrochemical biosensors or biofuel cells employing immobilized enzymes that will produce a targeted linear range, limit of detection and sensitivity. Such guidelines can be provided using analytical simulation tools that assess sensor feasibility prior to extensive development.

Biosensors and biofuel cell face increasing demand for selective and sensitive detection of different molecules for industrial, environmental and clinical applications [1]-[4]. There are many affordable alternatives to labora- 
tory techniques that require trained personnel, expensive equipment and possibly delayed response time. Electrochemical biosensors and biofuel cell especially desirable for use in field applications because of their compact design, ease of manufacture, real time response, sensitivity and selectivity [3]-[6]. They are used in many applications ranging from glucose detection to detection of neurotoxic agents [1] [6] [7]. Here we focus on biosensors and biofuel cell that employ immobilized enzymes and the electrochemical detection of the enzymatic reaction. Some important parameters that affect these goals are listed and include transport of the substrate and the product through the immobilized enzyme layer, oxidation/reduction kinetics at the electrode, enzyme activity and loading and operating conditions such as $\mathrm{pH}$ and temperature. Of these parameters optimizing the enzyme loading and activity has been a major challenge and it depends primarily on the enzyme immobilization method. Different methods such as chemical modification of the electrode surface, entrapment in a membrane and physical absorption are commonly used to create enzyme layers on electrodes [8].

A mathematical model considering reaction and diffusion processes in biofuel cell or biosensor, contains a system of non-linear partial differential equations. Numerical and analytical solutions to the reaction-diffusion equations have been presented for different cases by many authors [9]-[14]. Analytical solutions are available for limiting cases, whereas numerical solutions were used to determine and optimize a wide range of experimental parameters [15]. Many of the earlier studies have focused on optimizing glucose biosensors where the enzyme was entrapped in a redox hydrogel [16] [17]. Simple Michaelis-Menten kinetics was used to model the enzyme kinetics, and first order kinetics between the mediator and the electrode were assumed [9] [17]. The effects of experimental parameters on the response at steady state and during a transient were studied [12]. Especially the behavior of the glucose sensor in the diffusion limited regime was analyzed since this leads to an extended linear range [16] [18]. Substrate and product inhibition in an enzyme with first order reaction kinetics [19], diffusion through a semi-permeable outer membrane [20] [21] and data analysis to determine kinetic constants and enzyme activity [22] were also studied by different groups.

Sachin [23] used a finite difference method for electrochemical biosensors with an immobilized enzyme layer. Sachin described the general criteria using Michaelis-Menten rate equation and effect of gel thickness on the response of this biosensor. To our knowledge no rigorous analytical solutions for non-steady-state concentration and current have been reported. In this paper, we have derived the analytical expressions of concentration and current using a new approach of Homotopy perturbation method [24]-[27]. The result of the Equations (2)-(3) in immobilized enzyme system is relevant because its solution describes important applications such as biosensors, bioreactors, and biofuel cells, among others.

\section{Mathematical Formulation of the Problem}

The chemical reactions in the layer are

$$
E+S \leftrightarrow E S \rightarrow E+P
$$

where $E$ refers to the enzyme, $S$ is the substrate, $E S$ is a transitory complex assumed to be at a steady concentration, and $P$ is the product. The schematic of the system modeled in this study is shown in Figure 1 . An aqueous drop containing substrate $(S)$ is placed on the electrode with an immobilized enzyme layer. As the substrate diffuses through the enzyme layer it reacts with the enzyme to form the product $(P)$. The product then diffuses through the layer, and if it is electroactive, is oxidized or reduced at the electrode. When modeling this system, we used Michaelis-Menten equation to describe the kinetics within the enzyme layer and coupled it with Fick's law to describe the diffusion of the substrate and product as shown in Equations (2)-(3):

$$
\begin{aligned}
& \frac{\partial c_{S}}{\partial t}=D_{S} \frac{\partial^{2} c_{S}}{\partial z^{2}}-\frac{k_{c a t}[E] c_{S}}{c_{S}+K_{S}} \\
& \frac{\partial c_{P}}{\partial t}=D_{P} \frac{\partial^{2} c_{P}}{\partial z^{2}}+\frac{k_{c a t}[E] c_{S}}{c_{S}+K_{S}}
\end{aligned}
$$

where $c_{P}, c_{S}, D_{P}$ and $D_{S}$ represent the concentrations and diffusion coefficients of the product and the substrate, respectively. $k_{c a t}$ is the catalytic rate constant in the Michaelis-Menten mechanism, $[E]$ is enzyme loading, and $K_{S}$ is Michaelis constant for the substrate. In the above equations the initial and boundary conditions are given by 


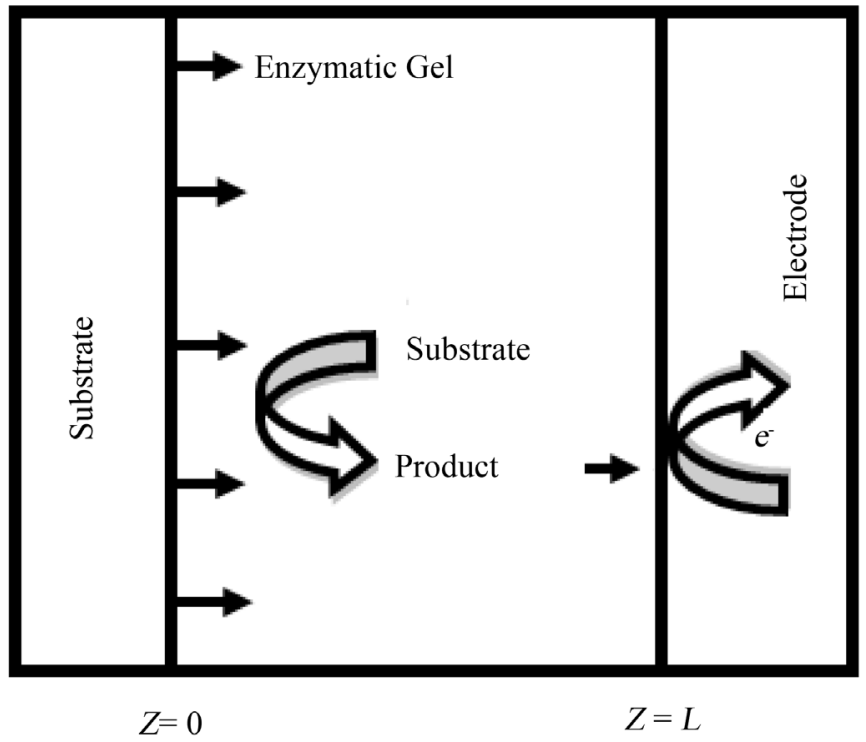

Figure 1. Schematic model of an enzyme-membrane electrodes.

$$
\begin{aligned}
& t=0, \quad 0 \leq z \leq L: \quad c_{s}=0, \quad c_{p}=0 \\
& t>0, z=0: \quad \frac{\partial c_{s}}{\partial z}=0, \quad \frac{\partial c_{p}}{\partial z}=0 \\
& t>0, z=L: \quad c_{s}=c_{\text {Sbulk }}, \quad c_{p}=0
\end{aligned}
$$

where $z$ is the distance from the electrode surface and $L$ is the enzyme layer thickness.

$c_{\text {Sbulk }}$ represents the concentration of substrate in bulk solution. Current $i$ occurring at the electrode surface due to reduction or oxidation of $P$ is given by

$$
i=n F D_{p}\left(\frac{\partial c_{p}}{\partial z}\right)_{z=d}
$$

Equations (2)-(3) were made dimensionless using the following dimensionless parameters:

$$
c_{s}^{*}=\frac{c_{s}}{K_{S}}, c_{p}^{*}=\frac{c_{P}}{K_{S}}, t^{*}=\frac{D_{S} t}{L^{2}}, z^{*}=\frac{z}{L}, r=\frac{D_{p}}{D_{s}}, \phi_{s}^{2}=\frac{k_{c a t}[E] L^{2}}{K_{S} D_{s}}
$$

The Equations (2)-(3) in dimensionless form becomes as follows:

$$
\begin{gathered}
\frac{\partial c_{s}^{*}}{\partial t}=\frac{\partial^{2} c_{s}^{*}}{\partial z^{* 2}}-\frac{\phi_{s}^{2} c_{s}^{*}}{c_{s}^{*}+1} \\
\frac{\partial c_{p}^{*}}{\partial t^{*}}=r \frac{\partial^{2} c_{p}^{*}}{\partial z^{* 2}}+\frac{\varphi_{s}^{2} c_{s}^{*}}{c_{s}^{*}+1}
\end{gathered}
$$

From the Equation (4), the initial and boundary conditions in dimensionless form are given by

$$
\begin{array}{ll}
t^{*}=0,0 \leq z^{*} \leq 1: & c_{s}^{*}=0, \quad c_{p}^{*}=0 \\
t^{*}>0, z^{*}=0: & \frac{\partial c_{s}^{*}}{\partial z^{*}}=0, \quad \frac{\partial c_{p}^{*}}{\partial z^{*}}=0 \\
t^{*}>0, z^{*}=1: & c_{s}^{*}=\frac{c_{\text {Sbulk }}}{K_{s}}=c_{s 0}, \quad c_{p}^{*}=0
\end{array}
$$

Dimensionless current density becomes 


$$
\psi=n F D_{p}\left(\frac{\partial c_{p}^{*}}{\partial z^{*}}\right)_{z^{*}=1}
$$

\section{General Analytical Expression of Concentration of Substrate and Product under Non-Steady State Condition Using Homotopy Perturbation Method (HPM)}

In recent days, HPM is often employed to solve several analytical problems. In addition, several groups demonstrated the efficiency and suitability of the HPM for solving nonlinear equations in electrochemical problems [28]-[31]. He et al. [24], used HPM to solve the Lighthill equation, the Duffing equation [25] and the Blasius equation [26]. HPM has also been used to solve non-linear boundary value problems [27], integral equation [32]-[34], Klein-Gordon and Sine-Gordon equations [35], Emden-Flower type equations [36] and several other problems. Laplace transform and Homotopy perturbation method are used to solve the non-linear differential Equations (7)-(8) (Appendix A). The analytical expressions of non-steady state concentrations are as follows:

$$
\begin{gathered}
c_{s}^{*}\left(z^{*}, t^{*}\right)=\frac{\cosh \left(\sqrt{a} z^{*}\right)}{\cosh (\sqrt{a})}-\pi \sum_{n=0}^{\infty} \frac{(-1)^{n}}{m}(2 n+1) \cos \left(\frac{2 n+1}{2} \pi z^{*}\right) \mathrm{e}^{-m t^{*}} \\
c_{p}^{*}\left(z^{*}, t^{*}\right)=1-\frac{\cosh \sqrt{a} z^{*}}{\cosh \sqrt{a}}-b \mathrm{e}^{-a t^{*} / b}\left(\frac{\cos \sqrt{(a r / b)} z^{*}}{\cos \sqrt{(a r / b)}}-\frac{\cosh \left(\sqrt{a(b-1) / b} z^{*}\right)}{\cosh (\sqrt{a(b-1) / b})}\right) \\
+\frac{4 a r}{\pi} \sum_{n=0}^{\infty} \frac{(-1)^{-n} \cos \left((2 n+1) \pi z^{*} / 2\right) \mathrm{e}^{-\left((2 n+1)^{2} \pi^{2} / 4 r\right) t^{*}}}{(2 n+1)\left[a-\left(b(2 n+1)^{2} \pi^{2} / 2 r\right)\right]} \\
-a \pi \sum_{n=0}^{\infty} \frac{(-1)^{-n} \cos \left((2 n+1) \pi z^{*} / 2\right)(2 n+1) \mathrm{e}^{-\left[a+(2 n+1)^{2} \pi^{2} / 4\right] t^{*}}}{\left[a+(2 n+1)^{2} \pi^{2} / 4\right]\left[a-b\left(a+(2 n+1)^{2} \pi^{2} / 4\right)\right]} \\
\text { where } m=a+\frac{\pi^{2}(2 n+1)^{2}}{4}, a=\frac{\phi_{s}^{2}}{1+c_{s 0}}, b=(1-r)
\end{gathered}
$$

Using (10) and (12), the current is given by

$$
\begin{aligned}
\frac{i}{n F D_{p}}= & \left(\frac{\partial c_{p}^{*}}{\partial z^{*}}\right)_{z^{*}=1}=\mid \sqrt{a b r} \tan (\sqrt{a r / b}) \mathrm{e}^{-a t^{*} / b}-\sqrt{a} \tanh (\sqrt{a})+\sqrt{a(b-1) b} \tanh \left(\sqrt{a(b-1) / b} \mathrm{e}^{-a t^{*} / b}\right. \\
& +\frac{8 r a \mathrm{e}^{-\pi^{2}(2 n+1)^{2} t^{*} / 4 r}}{4 r a-b \pi^{2}(2 n+1)^{2}}-8 a \pi^{2} \sum_{n=0}^{\infty} \frac{(2 n+1)^{2} \mathrm{e}^{-\left(4 a+\pi^{2}(2 n+1)^{2}\right)^{*} / 4}}{\left[4 a+\pi^{2}(2 n+1)^{2}\right]\left[4 a-b\left(4 a+\pi^{2}(2 n+1)^{2}\right)\right]} \mid
\end{aligned}
$$

When $t^{*} \rightarrow \infty$ (steady state), the above equation becomes

$$
\frac{i}{n F D_{p}}=|\sqrt{a} \tanh (\sqrt{a})|
$$

\section{Discussion}

Equations (11) (12) and (14) are the new and simple analytical expressions of concentrations of substrate, product and current respectively. To show the efficiency of our non-steady-state result, it is compared with numerical solution in Figure 2 \& Figure 3. Satisfactory agreement is noted. The SCILAB/MATLAB program is also given in Appendix B. Figure 2 shows the time-dependent normalized concentration profiles for the substrate $c_{s}^{*}$ in the enzyme membrane. Figures 2(a)-(c) show dimensionless concentration $c_{s}^{*}$ versus the dimensionless 


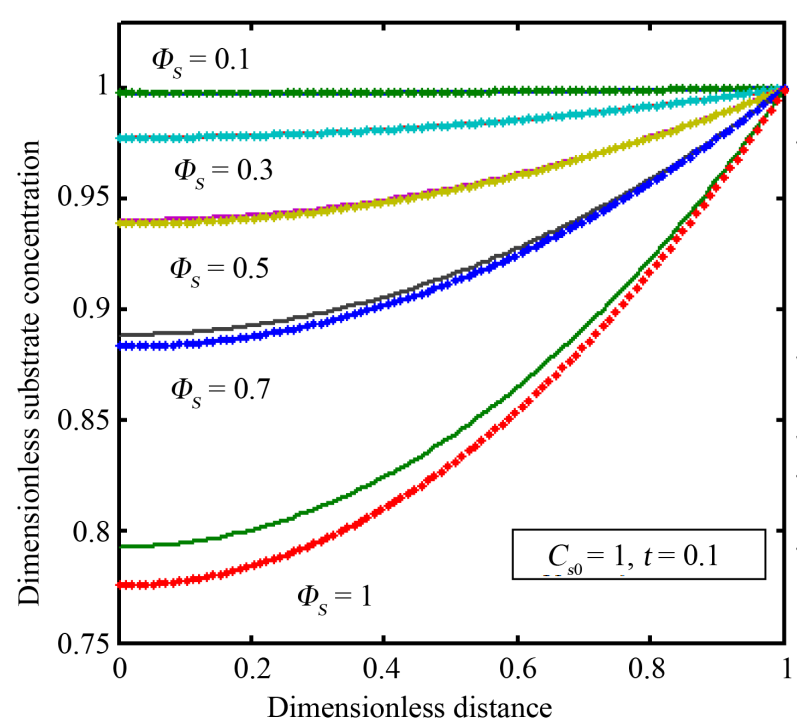

(a)

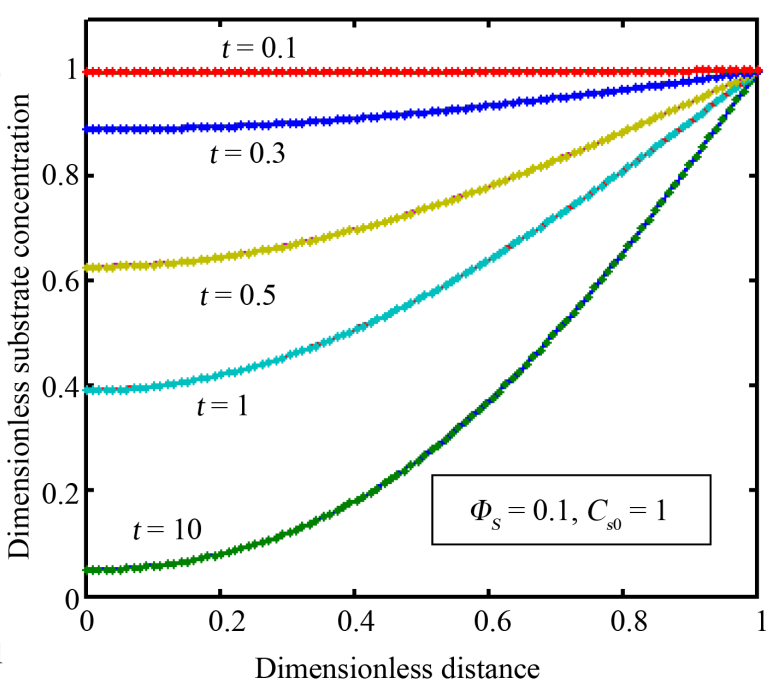

(b)



(c)

Figure 2. Dimensionless substrate concentration $c_{s}^{*}$ versus distance from electrode surface $z^{*}$ using Equation (11) for various values of parameters $\Phi_{s}, t$ and $c_{s 0}$.

distance $z^{*}$. The concentration of substrate $c_{s}^{*}$ depends upon the dimensionless parameter " $a$ ". The dimensionless parameter " $a$ " depends upon $\phi_{s}$ and $c_{s 0}$. When Thiele modulus $\phi_{s}$ is small, the kinetics dominate and the uptake of the substrate are kinetically controlled. From Figure 2(a), it is evident that the value of the substrate concentration $c_{s}^{*}$ decreases when the Thiele modulus $\phi_{s}$ increases or Figure 2(b) illustrates that, when $t$ increases, the concentration of the substrate decreases. It is obvious from Figure 2(c) that when initial substrate concentration $c_{s 0}$ increases, the concentration of substrate also decreases.

The normalized concentration of the product $c_{p}^{*}$ for various values of Thiele modulus $\phi_{s}$, time $t$ and ratio of diffusion coefficient is plotted in Figures 3(a)-(c). From the Figure 3(a) \& Figure 3(b), it is inferred that the normalized concentration product increases with the decrease in the value of $\phi_{s}$ and time $t$. The product concentration is increases when the ratio of diffusion coefficient decreases as shown in Figure 3(c).

The value of current $i$ increases slightly when the Thiele modulus $\phi_{s}$ increases or electrode thickness increases as shown in Figure 4(a). From Figure 4(b), it is inferred that the ratio of diffusion coefficient $r$ increases the current density is decreases. The current density increases as initial substrate concentration $c_{s 0}$ 


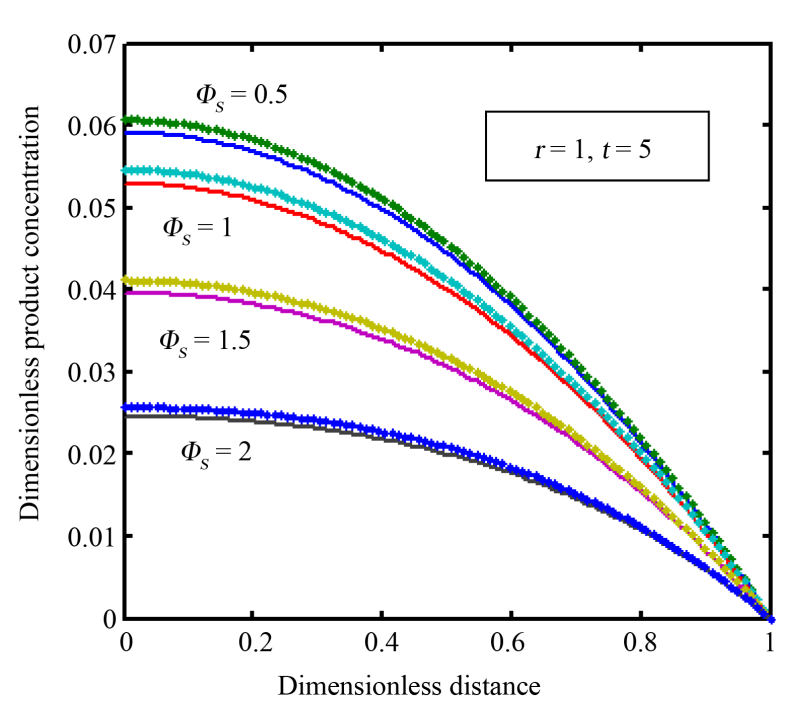

(a)

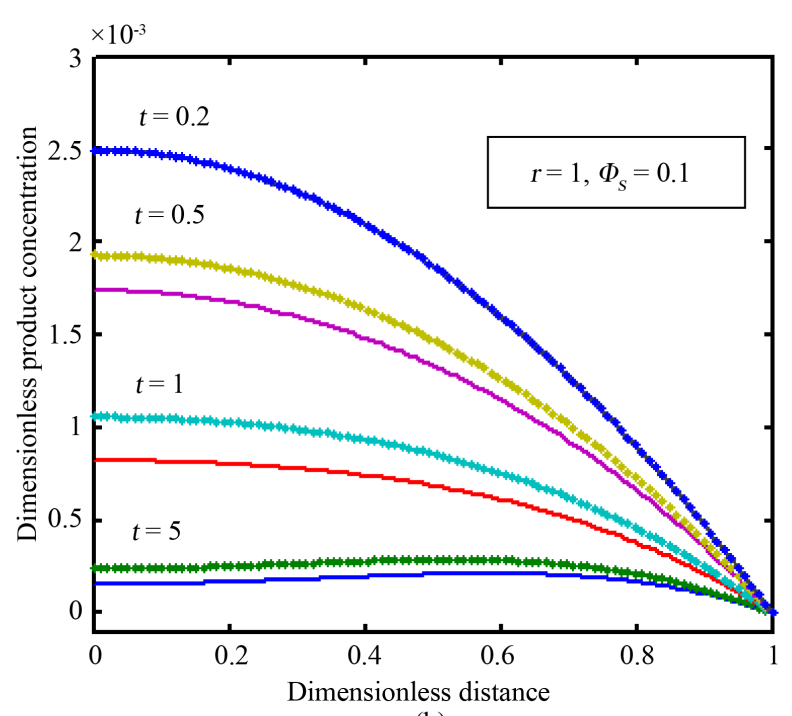

(b)

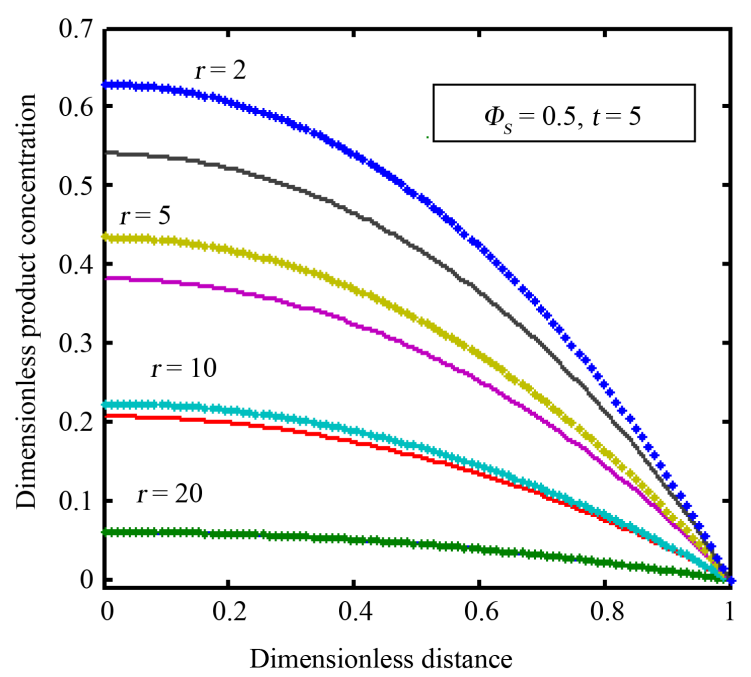

(c)

Figure 3. Dimensionless product concentration $c_{p}^{*}$ versus distance from electrode surface $z^{*}$ using Equation (12) for various values of parameters $\Phi_{s}, t$ and $r$.

decreases.

\section{Estimation of Kinetic Parameters}

The current is dependent upon the parameters Thiele module $\phi_{s}$ and initial substrate concentration $c_{s 0}$. When $r=1$, (or $D_{p}=D_{s}$ ) Equation (15) can be written as

$$
\frac{\phi_{s}^{2}}{1+c_{s 0}}=\left[\tanh ^{-1}\left(-i / n F D_{p}\right)\right]^{2}
$$

Substituting the value of $\mu$ and $k$ in the above equation, we get

$$
\left[\tanh ^{-1}\left(-i / n F D_{p}\right)\right]^{-2}=\left(\frac{D_{s}}{k_{\text {cat }}[E] L^{2}}\right) C_{\text {Sbulk }}+\frac{K_{S} D_{s}}{k_{\text {cat }}[E] L^{2}}
$$




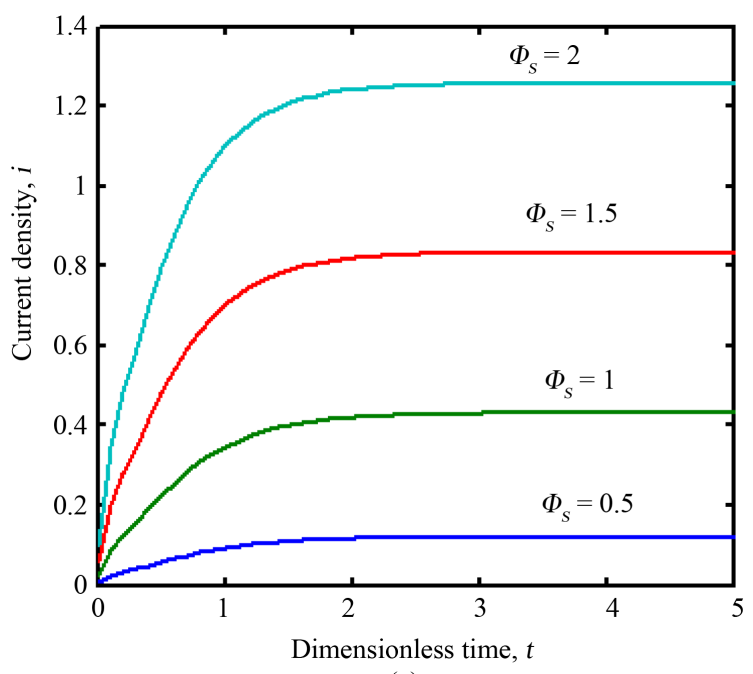

(a)



(b)

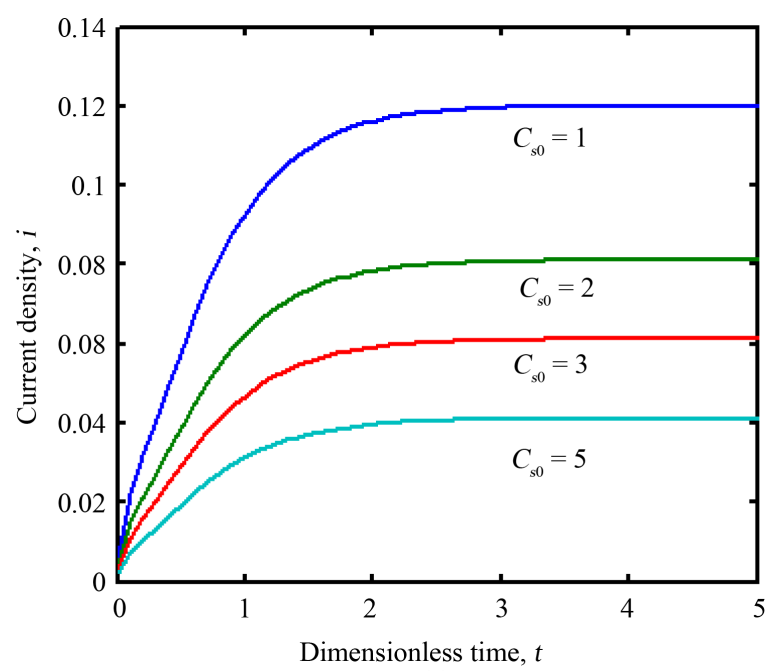

(c)

Figure 4. Dimensionless current density $i / n F D_{p}$ versus time $t$ using Equation (14) for various values of parameters $\Phi_{s}, c_{s 0}$ and $r$.

The plot of $\left[\tanh ^{-1}\left(-i / n F D_{p}\right)\right]^{-2}$ versus $C_{\text {Sbulk }}$ gives the slope $\left(D_{s} / k_{\text {cat }}[E] L^{2}\right)$ and intercept $\left(K_{s} D_{s} / k_{c a t}[E] L^{2}\right)$ as shown in Figure 5. From these plots, we can obtain the value of kinetic parameters $K_{S}$ and $\left(D_{s} / k_{c a t}\right)$.

\section{Conclusion}

The theoretical behavior of biofuel cell/biosensor was analyzed. The coupled time dependent non-steady state non-linear diffusion equations in biosensor or biofuel cell have been solved analytically and numerically. These analytical results will be used in determining the kinetic characteristics of the biofuel cell or biosensor. The analytical expressions for substrate, product concentration and transient current response are obtained using the method of Laplace transformation and HPM. A good agreement with numerical simulation data is noticed. Concentration of substrate, product and current depends upon Thiele modulus $\phi_{s}$ and initial concentration of substrate which is discussed in this communication. Evaluation of kinetic parametr from the response of the steady-state current is also completely discussed. The theoretical model presented here can be used for the optimization of the design of the biosensor. 


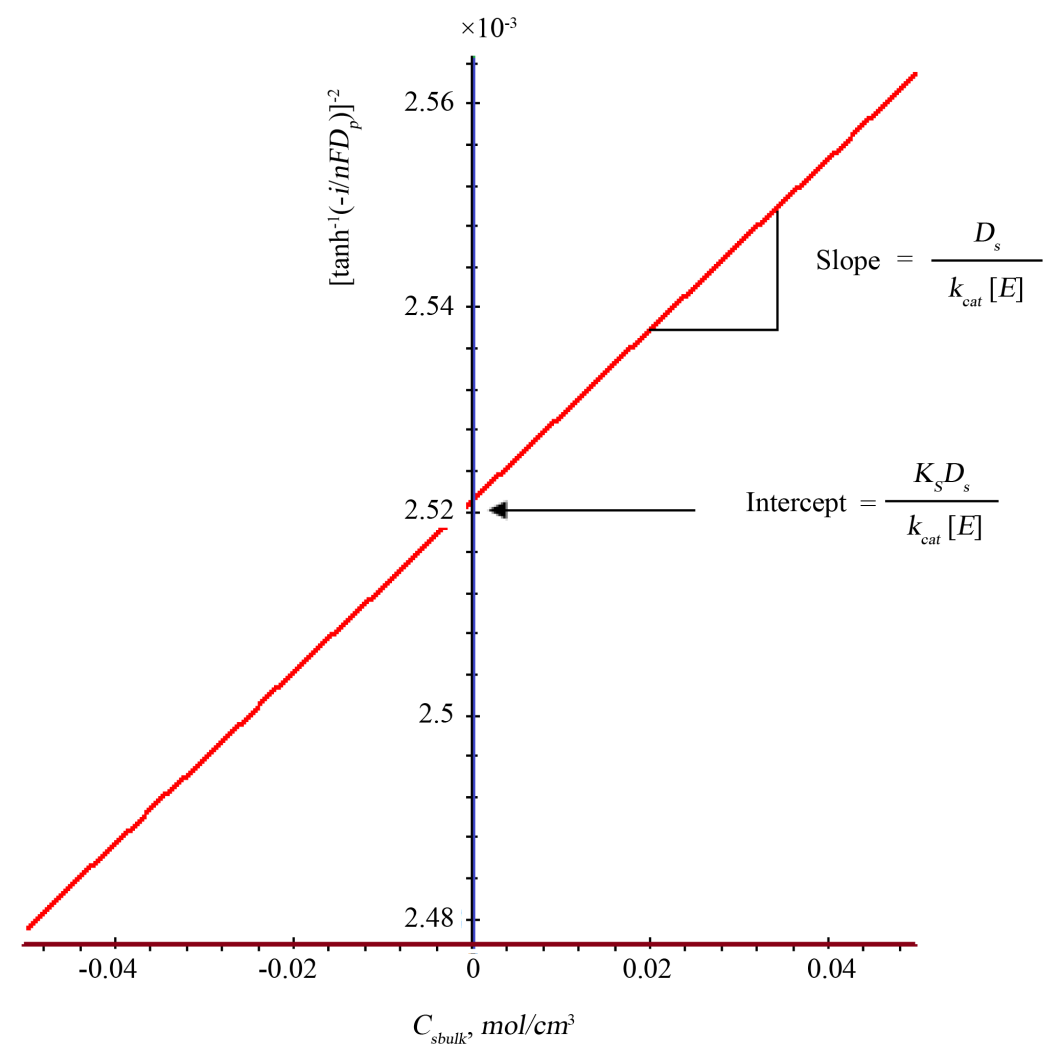

Figure 5. A plot of $\tan ^{-1}\left(i / n F D_{p}\right)^{-2}$ versus initial substrate concentration $C_{\text {Sbulk }}$ using Equation (17) to estimate the kinetic parameters.

\section{Acknowledgements}

This work is supported by the Department of Science and Technology (DST) (No. SB/SI/PC-50/2012), Government of India. The authors are thankful to Shri. S. Natanagopal, Secretary, The Madura College Board and Dr. R. Murali, Principal, Mr. S. Muthukumar, Head of the Department, Department of Mathematics, The Madura College (Autonomous), Madurai, Tamilnadu, India for their constant encouragement.

\section{References}

[1] Wang, J., Krause, R., Block, K., Musameh, M., Mulchandani, A. and Schöning, M.J. (2003) Flow Injection Amperometric Detection of OP Nerve Agents Based on an Organophosphorus-Hydrolase Biosensor Detector. Biosensors and Bioelectronics, 18, 255-260. http://dx.doi.org/10.1016/S0956-5663(02)00178-1

[2] Dennison, M.J., Hall, J.M. and Turner, A.P.F. (1996) Direct Monitoring of Formaldehyde Vapour and Detection of Ethanol Vapour Using Dehydrogenase-Based Biosensors. Analyst, 121, 1769-1773. http://dx.doi.org/10.1039/an9962101769

[3] Wu, J., Cropek, D.M., West, A.C. and Banta, S. (2010) Development of a Troponin I Biosensor Using a Peptide Obtained through Phage Display. Analytical Chemistry, 82, 8235-8243. http://dx.doi.org/10.1021/ac101657h

[4] Chen, X.J., West, A.C., Cropek, D.M. and Banta, S. (2008) Detection of the Superoxide Radical Anion Using Various Alkanethiol Monolayers and Immobilized Cytochrome c. Analytical Chemistry, 80, 9622-9629. http://dx.doi.org/10.1021/ac800796b

[5] Lei, Y., Mulchandani, P., Wang, J., Chen, W. and Mulchandani, A. (2005) A Highly Sensitive and Selective Amperometric Microbial Biosensor for Direct Determination of $p$-Nitrophenyl-Substituted Organophosphate Nerve Agents. Environmental Science \& Technology, 39, 8853-8857 http://dx.doi.org/10.1021/es050720b

[6] Sahin, A., Dooley, K., Cropek, D.M., West, A.C. and Banta, S. (2011) A Dual Enzyme Electrochemical Assay for the Detection of Organophosphorus Compounds Using Organophosphorus Hydrolase and Horseradish Peroxidase. Sensors and Actuators B: Chemical, 158, 353-360. http://dx.doi.org/10.1016/j.snb.2011.06.034 
[7] Cass, A.E.G., Davis, G., Francis, G.D., Hill, H.A.O., Aston, W.J., Higgins, I.J., Plotkin, E.V., Scott, L.D.L. and Turner, A.P.F. (1984) Ferrocene-Mediated Enzyme Electrode for Amperometric Determination of Glucose. Analytical Chemistry, 56, 667-671. http://dx.doi.org/10.1021/ac00268a018

[8] Sheldon, R.A. (2007) Enzyme Immobilization: The Quest for Optimum Performance. Advanced Synthesis \& Catalysis, 349, 1289-1307. http://dx.doi.org/10.1002/adsc.200700082

[9] Bartlett, P.N. and Pratt, K.F.E. (1995) Theoretical Treatment of Diffusion and Kinetics in Amperometric Immobilized Enzyme Electrodes Part I: Redox Mediator Entrapped within the Film. Journal of Electroanalytical Chemistry, 397, 61-78. http://dx.doi.org/10.1016/0022-0728(95)04236-7

[10] Flexer, V., Pratt, K.F.E., Garay, F., Bartlett, P.N. and Calvo, E.J. (2008) Relaxation and Simplex Mathematical Algorithms Applied to the Study of Steady-State Electrochemical Responses of Immobilized Enzyme Biosensors: Comparison with Experiments. Journal of Electroanalytical Chemistry, 616, 87-98. http://dx.doi.org/10.1016/j.jelechem.2008.01.006

[11] Lyons, M. (2006) Modelling the Transport and Kinetics of Electroenzymes at the Electrode/Solution Interface. Sensors, 6, 1765-1790. http://dx.doi.org/10.3390/s6121765

[12] Kartono, A., Sulistan, E. and Mamat, M. (2010) The Numerical Analysis of Enzyme Membrane Thickness on the Response of Amperometric Biosensor. Applied Mathematical Sciences, 4, 1299-1308.

[13] Baronas, R., Ivanauskas, F. and Kulys, J. (2009) Mathematical Modeling of Biosensors: An Introduction for Chemists and Mathematicians. Springer, Dordrecht.

[14] Shunmugham, L. and Rajendran, L. (2013) Analytical Expressions for Steady-State Concentrations of Substrate and Oxidized and Reduced Mediator in an Amperometric Biosensor. International Journal of Electrochemistry, 2013, 1-12. http://dx.doi.org/10.1155/2013/812856

[15] Meena, A. and Rajendran, L. (2010) Mathematical Modeling of Amperometric and Potentiometric Biosensors and System of Non-Linear Equations-Homotopy Perturbation Approach. Journal of Electroanalytical Chemistry, 644, 5059. http://dx.doi.org/10.1016/j.jelechem.2010.03.027

[16] Cambiaso, A., Delfino, L., Grattarola, M., Verreschi, G., Ashworth, D., Maines, A. and Vadgama, P. (1996) Modelling and Simulation of a Diffusion Limited Glucose Biosensor. Sensors and Actuators B: Chemical, 33, 203-207. http://dx.doi.org/10.1016/0925-4005(96)80099-2

[17] Mell, L.D. and Maloy, J.T. (1975) Model for the Amperometric Enzyme Electrode Obtained through Digital Simulation and Applied to the Immobilized Glucose Oxidase System. Analytical Chemistry, 47, 299-307. http://dx.doi.org/10.1021/ac60352a006

[18] Mell, L.D. and Maloy, J.T. (1976) Amperometric Response Enhancement of the Immobilized Glucose Oxidase Enzyme Electrode. Analytical Chemistry, 48, 1597-1601. http://dx.doi.org/10.1021/ac50005a045

[19] Šimelevičius, D. and Baronas, R. (2010) Computational Modelling of Amperometric Biosensors in the Case of Substrate and Product Inhibition. Journal of Mathematical Chemistry, 47, 430-445. http://dx.doi.org/10.1007/s10910-009-9581-x

[20] Puida, M., Ivanauskas, F. and Laurinavičius, V. (2010) Mathematical Modeling of the Action of Biosensor Possessing Variable Parameters. Journal of Mathematical Chemistry, 47, 191-200. http://dx.doi.org/10.1007/s10910-009-9541-5

[21] Schulmeister, T. and Pfeiffer, D. (1993) Mathematical Modelling of Amperometric Enzyme Electrodes with Perforated Membranes. Biosensors and Bioelectronics, 8, 75-79. http://dx.doi.org/10.1016/0956-5663(93)80055-T

[22] Rinken, T. (2003) Determination of Kinetic Constants and Enzyme Activity from a Biosensor Transient Signal. Analytical Letters, 36, 1535-1545. http://dx.doi.org/10.1081/AL-120021535

[23] Sachin, A. (2012) Development of Electrochemical Methods for Detection of Pesticides and Biofuel Production. Columbia University, New York.

[24] He, J.H. (1999) Homotopy Perturbation Technique. Computer Methods in Applied Mechanics and Engineering, 178, 257-262. http://dx.doi.org/10.1016/S0045-7825(99)00018-3

[25] He, J.H. (2003) Homotopy Perturbation Method: A New Nonlinear Analytical Technique. Applied Mathematics and Computation, 135, 73-79. http://dx.doi.org/10.1016/S0096-3003(01)00312-5

[26] He, J.H. (2003) A Simple Perturbation Approach to Blasius Equation. Applied Mathematics and Computation, 140, 217-222. http://dx.doi.org/10.1016/S0096-3003(02)00189-3

[27] He, J.H. (2006) Homotopy Perturbation Method for Solving Boundary Value Problems. Physics Letters A, 350, 87-88. http://dx.doi.org/10.1016/j.physleta.2005.10.005

[28] Ghori, Q.K., Ahmed, M. and Siddiqui, A.M. (2007) Application of Homotopy Perturbation Method to Squeezing Flow of a Newtonian Fluid. International Journal of Nonlinear Sciences and Numerical Simulation, 8, 179-184. http://dx.doi.org/10.1515/ijnsns.2007.8.2.179 
[29] Ozis, T. and Yildirim, A. (2007) Relation of a Nonlinear Oscillator with Discontinuities. International Journal of Nonlinear Sciences and Numerical Simulation, 8, 243-248. http://dx.doi.org/10.1515/IJNSNS.2007.8.2.243

[30] Li, S.J. and Liu, Y.X. (2006) An Improved Approach to Nonlinear Dynamical System Identification Using PID Neural Networks. International Journal of Nonlinear Sciences and Numerical Simulation, 7, 177-182. http://dx.doi.org/10.1515/IJNSNS.2006.7.2.177

[31] Mousa, M.M. and Ragab, S.F. (2008) Application of the Homotopy Perturbation Method to Linear and Nonlinear Schrodinger Equations. Zeitschrift für Naturforschung A, 63, 140-144. http://dx.doi.org/10.1515/zna-2008-3-404

[32] Golbabai, A. and Keramati, B. (2008) Modified Homotopy Perturbation Method for Solving Fredholm Integral Equations. Chaos, Solitons \& Fractals, 37, 1528-1537. http://dx.doi.org/10.1016/j.chaos.2006.10.037

[33] Ghasemi, M., Kajani, T.M. and Babolian, E. (2007) Numerical Solutions of the Nonlinear Volterra-Fredholm Integral Equations by Using Homotopy Perturbation Method. Applied Mathematics and Computation, 188, 446-449. http://dx.doi.org/10.1016/j.amc.2006.10.015

[34] Biazar, J. and Ghazvini, H. (2009) He’s Homotopy Perturbation Method for Solving System of Volterra Integral Equations of the Second Kind. Chaos, Solitons \& Fractals, 39, 770-777. http://dx.doi.org/10.1016/j.chaos.2007.01.108

[35] Odibat, Z., Momani, S., Odibat, Z. and Momani, S. (2007) A Reliable Treatment of Homotopy Perturbation Method for Klein-Gordon Equations. Physics Letters A, 365, 351-357. http://dx.doi.org/10.1016/j.physleta.2007.01.064

[36] Chowdhury, M.S.H. and Hashim, I. (2007) Solutions of Time-Dependent Emden-Fowler Type Equations by Homotopy-Perturbation Method. Physics Letters A, 368, 305-313. http://dx.doi.org/10.1016/j.physleta.2007.04.020

\section{Nomenclature}

\begin{tabular}{|c|c|}
\hline Symbols & Symbols \\
\hline$\phi_{s}$ & Dimensionless ratio of the enzymatic reaction rate to diffusion rate of product, (none) \\
\hline$\phi_{p}$ & Dimensionless ratio of the enzymatic reaction rate to diffusion rate of substrate,(none) \\
\hline$a, b$ & Dimensionless parameters (none) \\
\hline$c_{p}$ & Concentration of product, $\mathrm{mol} / \mathrm{cm}^{3}$ \\
\hline$c_{p}^{*}$ & Dimensionless product concentration, (none) \\
\hline$c_{s}$ & Concentration of substrate, $\mathrm{mol} / \mathrm{cm}^{3}$ \\
\hline$C_{s}^{*}$ & Dimensionless substrate concentration, (none) \\
\hline$C_{\text {sbulk }}$ & Concentration of substrate in bulk solution, $\mathrm{mol} / \mathrm{cm}^{3}$ \\
\hline$C_{\text {sbulk }}$ & Dimensionless concentration of substrate in bulk solution, (none) \\
\hline$D_{p}^{*}$ & Diffusion coefficient of product, $\mathrm{cm}^{2} / \mathrm{s}$ \\
\hline$D_{s}$ & Diffusion coefficient of substrate, $\mathrm{cm}^{2} / \mathrm{s}$ \\
\hline$F$ & Faraday’s constant, C/mol \\
\hline$I$ & Current density, A/cm² \\
\hline$K_{S}$ & Michaelis constant for reactant, mM \\
\hline$k_{\text {cat }}$ & Catalytic rate constant in Michaelis-Menten mechanism, $\mathrm{s}^{-1}$ \\
\hline$L$ & Thickness of enzyme layer, cm \\
\hline$n$ & Number of electrons transferred in reaction, (none) \\
\hline$r$ & Ratio of diffusion coefficients, (none) \\
\hline$t$ & Time, s \\
\hline$t^{*}$ & Dimensionless time, (none) \\
\hline$V_{\max }$ & Product of the kinetic parameter and enzyme loading, mM/s \\
\hline$Z$ & Distance from the electrode surface, $\mathrm{cm}$ \\
\hline$z^{*}$ & Dimensionless distance from the electrode surface, (none) \\
\hline
\end{tabular}




\section{Appendix A}

\section{Solution of Equations (7) and (8) Using Complex Inversion Formula}

In this appendix we indicate how Equations (11) and (12) are derived, by solving a differential equation of second order with constant coefficients by using new homotopy approach and Laplace transform in Equations (7) and (8), and the boundary conditions. The obtained solution of the Equation (7) as

$$
\overline{c_{s}^{*}}=\frac{\cosh \sqrt{s+a} z^{*}}{s \cosh \sqrt{s+a}}
$$

In this appendix we indicate how Equation (A1) may be inverted using the complex inversion formula. If $\bar{y}(s)$ represents the Laplace transform of a function $y(\tau)$, then according to the complex inversion formula we can state that

$$
y(\tau)=\frac{1}{2 \pi \int_{c-i \infty}^{c+i \infty} \exp [s \tau] \bar{y}(s) \mathrm{d} s}=\frac{1}{2 \pi i} \oint_{c} \exp [s \tau] \bar{y}(s) \mathrm{d} s
$$

where the integration in Equation (A2) is to be performed along a line $s=c$ in the complex plane where $s=x+i y$. The real number $c$ is chosen such that $s=c$ lies to the right of all the singularities, but is otherwise assumed to be arbitrary. In practice, the integral is evaluated by considering the contour integral presented on the right-hand side of Equation (A2), which is then evaluated using the so-called Bromwich contour. The contour integral is then evaluated using the residue theorem which states for any analytic function $F(z)$

$$
\oint_{c} F(z) \mathrm{d} z=2 \pi i \sum_{n} \operatorname{Re} s[F(z)]_{z=z_{0}}
$$

where the residues are computed at the poles of the function $F(z)$. Hence from Equation (A3), we note that

$$
y(\tau)=\sum_{n} \operatorname{Res}[\exp [s \tau] \bar{y}(s)]_{s=s_{0}}
$$

From the theory of complex variables we can show that the residue of a function $F(z)$ at a simple pole at $\mathrm{Z}=a$ is given by

$$
\operatorname{Re} s[F(z)]_{z=a}=\lim _{z \rightarrow a}\{(z-a) F(z)\}
$$

Hence in order to invert Equation (A1), we need to evaluate

$$
\operatorname{Res}\left[\frac{\cosh (\sqrt{s+a}) z^{*}}{s \cosh (\sqrt{s+a})}\right]
$$

The poles are obtained from $s \cosh \sqrt{s+a}=0$. Hence there is a simple pole at $s=0$ and there are infinitely many poles given by the solution of the equation $\cosh \sqrt{s+a}=0$ and

$$
\text { so } s_{n}=\frac{-\pi^{2}(2 n+1)^{2}-4 a}{4} \text { where } n=0,1,2, \cdots
$$

Hence we note that

$$
c_{s}^{*}\left(z^{*}, t\right)=\operatorname{Re} s[s \cosh (\sqrt{s+a})]_{s=0}+\operatorname{Re} s[s \cosh (\sqrt{s+a})]_{s=s_{n}}
$$

The first residue in Equation (A6) is given by

$$
\begin{aligned}
\operatorname{Re} s[s \cosh (\sqrt{s+a})]_{s=0} & =\lim _{s \rightarrow 0}\left[\frac{\exp (s \tau) \cosh (\sqrt{s+a}) X}{s \cosh (\sqrt{s+a})}\right] \\
& =\frac{\cosh \sqrt{a} z^{*}}{\cosh \sqrt{a}}
\end{aligned}
$$


The second residue in Equation (A6) is given by

$$
\begin{aligned}
\operatorname{Res}[s \cosh (\sqrt{s+a})]_{s=s_{n}} & =\lim _{s \rightarrow s_{n}}\left[\frac{\exp (s t) \cosh (\sqrt{s+a}) z^{*}}{s \cosh (\sqrt{s+a})}\right] \\
& =\lim _{s \rightarrow s_{n}}\left[\frac{\exp (s t) \cosh (\sqrt{s+a}) z^{*}}{s \frac{\mathrm{d}}{\mathrm{d} s} \cosh (\sqrt{s+a})}\right] \\
& =\sum_{n=0}^{\infty}\left[\frac{(-1)^{n} \pi(2 n+1) \mathrm{e}^{-m t} \operatorname{os}\left[(2 n+1) \pi z^{*} / 2\right]}{m}\right]
\end{aligned}
$$

where $m$ is defined as in Equation (13). Here we used $\cosh (i \theta)=\cos (\theta)$ and $\sinh (i \theta)=i \sin (\theta)$. From (A6), (A7) and (A8) we conclude that

$$
c_{s}^{*}\left(z^{*}, t\right)=\frac{\cosh \sqrt{a} z^{*}}{\cosh \sqrt{a}}-\sum_{n=0}^{\infty}\left[\frac{(-1)^{n} \pi(2 n+1) \mathrm{e}^{-m t} \cos \left[(2 n+1) \pi z^{*} / 2\right]}{m}\right]
$$

Similarly we can invert Equation (8) by using complex inversion formula.

\section{Appendix B}

Scilab/Matlab Program to Find the Numerical Solution of Equations (7) and (8)

function see5

$\mathrm{m}=0$;

$\mathrm{x}=$ linspace $(0,1)$;

$\mathrm{t}=$ linspace $(0,5)$;

sol=pdepe(m,@pdex4pde,@pdex4ic,@pdex4bc,x,t);

u1 = sol(:,:,1);

u2 = sol(:,:,2);

\%figure

$\% \operatorname{plot}(\mathrm{x}, \mathrm{u} 1(\mathrm{end}, \mathrm{)}))$

$\%$ title('u1(x,t)')

\%xlabel('Distance $\mathrm{x}$ ')

\%ylabel('time ')

$\%-$

figure

plot(x,u2(end,:))

title('u2(x,t)')

xlabel('Distance $\mathrm{x}$ ')

ylabel('u2(x,2)')

$\%$ -

function $[\mathrm{c}, \mathrm{f}, \mathrm{s}]=\operatorname{pdex} 4 \mathrm{pde}(\mathrm{x}, \mathrm{t}, \mathrm{u}, \mathrm{DuDx})$

$\mathrm{r}=1$;

$\mathrm{C}=[1 ; \mathrm{r}]$;

$\mathrm{f}=[1 ; 1] *$ DuDx;

$\mathrm{e}=0.5$;

$\mathrm{F}=\left(\mathrm{e}^{\wedge} 2\right)^{*} \mathrm{u}(1) /(1+\mathrm{u}(1)) ;$

$\mathrm{s}=[-\mathrm{F}, \mathrm{F}]$;

$\%$

function $\mathrm{u} 0=\operatorname{pdex} 4 \mathrm{ic}(\mathrm{x})$

$\mathrm{u} 0=[0 ; 0]$; 
$\%$

function [pl,ql,pr,qr]=pdex4bc(xl,ul,xr,ur,t)

$\mathrm{pl}=[0 ; 0]$;

$\mathrm{ql}=[1 ; 1] ;$

$\mathrm{pr}=[\operatorname{ur}(1)-1 ; \operatorname{ur}(2)-0]$;

$\mathrm{qr}=[0 ; 0]$; 